\title{
Electroactive shape memory performance of polyurethane composite having homogeneously dispersed and covalently crosslinked carbon nanotubes
}

Yong Chae Jung, ${ }^{a}$ Hye Jin Yoo, ${ }^{\mathrm{b}}$ Yoong Ahm Kim, ${ }^{* a}$ Jae Whan Cho, ${ }^{* b}$ and Morinobu Endo $^{\mathrm{a}}$

${ }^{a}$ Faculty of Engineering, Shinshu University, Wakasato, Nagano-shi 380-8553, Japan

${ }^{b}$ Department of Textile Engineering, Konkuk University, Seoul 143-710, Korea

\begin{abstract}
The electroactive shape memory of carbon nanotube-filled polyurethane composites, prepared by conventional blending, in-situ and cross-linking polymerization, is studied in terms of the dispersion of the tubes. The covalently bonded tubes are homogeneously dispersed within the polyurethane by introducing carboxyl groups on the sidewall of the tubes and selecting a cross-linking polymerization method. The resultant composites, which have $92 \%$ shape retention and $95 \%$ shape recovery, are expected to be used as preferential materials in various actuators.

* Corresponding authors. Fax: +81-26-269-5656 (Y. A. Kim), and +82-2-457-8895 (J. W. Cho). E-mail addresses: yak@endomoribu.shinshu-u.ac.jp (Y. A. Kim), and jwcho@konkuk.ac.kr (J. W. Cho)
\end{abstract}




\section{Introduction}

Carbon nanotubes have been considered as an ideal reinforcing agent for high-performance polymeric composites due to their excellent structural, mechanical and electrical properties [1, 2]. However, the homogeneous dispersion of the tubes within polymer matrices still presents a challenge. Various methods have been examined in efforts to overcome this problem, such as mechanical mixing [3], chemical functionalization of the tubes [4-6], and the utilization of organic [7, 8] and polymeric surfactants [9] via non-covalent interactions. An in-situ polymerization [10], where nanotubes are incorporated into the prepolymer during the polymerization process, has been utilized to provide carbon nanotubes-reinforced composites with enhanced mechanical properties. On the other hand, the functionalization of carbon nanotubes is also very effective in preparing soluble carbon nanotubes by generating structural defects in strong acid [11, 12], and inducing a chemical reaction between alkyl groups and azide moieties [13], and $\pi-\pi$ interaction with electron-rich molecules $[6,14]$.

This work is focused on the effects of the controlled dispersion structure of the tubes on the mechanical and electroactive memory of conductive polymeric composites. Carbon nanotubes generally aggregate to form bundles, and their dispersion structure strongly depends on the physical and chemical interactions between the tubes and the surrounding solvents or polymers. In this study, we have attempted to control the dispersion structure of the tubes in polymer matrix via in-situ and cross-linking polymerization $[15,16]$. A comparison of these two methods might be useful for finding an effective way of controlling the dispersion structures of the tubes within the 
polymer matrix as well as the electroactive memory of electrically conductive polymeric composites. In order to achieve this goal, we chose a polyurethane block copolymer as the polymer matrix due to its excellent shape memory, which is caused by the phase separation between hard and soft segments and thermo-responsive transitions corresponding to soft segments [17], together with acid-treated multi-walled carbon nanotubes (MWCNTs) as the reinforcing filler rather than carbon black [18]. Subsequently, we prepared polyurethane composites reinforced with carbon nanotubes using three different polymerization methods (namely conventional blending, in-situ, and cross-linking polymerization), and performed a comparative study of the dispersion structure of the carbon nanotubes and their effects on the mechanical and electroactive shape memory of three types of polyurethane composites filled with nanotubes.

\section{Experimental}

MWCNTs (Iljin Nano Tech) with a diameter of 10-20 nm and an average length of 20 $\mu \mathrm{m}$ were used after being subjected to chemical modification in a mixture of $\mathrm{HNO}_{3} / \mathrm{H}_{2} \mathrm{SO}_{4}\left(1: 3\right.$ molar ratio) at $90^{\circ} \mathrm{C}$ for $10 \mathrm{~min}$. Poly( $\varepsilon$-carprolactone)diol (PCL, Solvay Co., MW=3,000 g/mol), 4,4`-methylene bis(phenylisocyanate) (MDI, Junsei Chemical), and 1,4-butandiol (BD, Ducksan Chemical) were used for the synthesis of PU. For the in-situ polymerization, the specified weight fractions of the acid-treated MWCNTs were dispersed in PCL. After that, MDI was added to this mixture to obtain prepolymers by the reaction between the $-\mathrm{OH}$ groups of the polyol and the $-\mathrm{NCO}$ groups of MDI at $80^{\circ} \mathrm{C}$ for $90 \mathrm{~min}$. In this case, most of the reaction between $-\mathrm{OH}$ and 
-NCO occurs since the carboxylic groups in acid-treated MWCNTs have relatively low reactivity in comparison to the reaction between PCL and MDI molecules. Finally, BD was added to the prepolymer in order to obtain nanotube-filled polyurethane composites by in-situ polymerization. Regarding the cross-linking polymerized composites, the prepolymer was prepared from a reaction between MDI and PCL under the same conditions as the in-situ polymerization, after which a calculated amount of tubes was added to the prepolymer at $110^{\circ} \mathrm{C}$ and the mixture was left for $150 \mathrm{~min}$ in order for covalent bonds to form between the $-\mathrm{OH}$ groups of the acid-treated MWCNTs and the $-\mathrm{NCO}$ groups of the prepolymer. As a comparative sample, we have prepared the conventional composites by blending carbon nanotubes with polyurethane using a mixed solvent consisting of tetrahydrofuran (THF) and dimethylformamide (DMF) $(1 / 1 \mathrm{w} / \mathrm{w})$. In all cases, the added amount of tubes with respect to polyurethane was fixed at $4 \mathrm{wt} \%$. We have prepared composite films having a thickness of ca. $150 \mu \mathrm{m}$ by casting nanotube/polyurethane emulsions on a clean, flat-base Petri dish and drying in an oven.

Raman spectroscopy (Kaiser, $633 \mathrm{~nm}$ ) was used to measure the structural changes of the carbon nanotubes resulting from the acid treatment. X-ray photoelectron spectroscopy (XPS) (ESCA2000) was adopted in order to measure the surface composition of the nanotubes. Transmission electro microscopy (TEM) measurements were carried out in order to observe the morphology of the nanotubes by using TEM-2000FX2 (JEOL), while the surface topology and the current images of the spin-coated samples were obtained by contact-mode atomic force microscopy (AFM) (Seiko Instruments Inc., SPA-300HV + SPI-3800N). Finally, the shape memory test of the composite films was carried out within a controlled thermal chamber, as described 
in our previous report [17]. The electroactive shape memory properties of shape recovery and shape retention were evaluated by applying constant electric voltage while monitoring the temperature and the length of the samples [16].

\section{Results and Discussion}

The dispersed state (or structure) of the nanotubes within the polymer matrix is influenced by the surface properties of the carbon nanotubes. In order to confirm the modification of the surface properties of the tubes resulting from the acid treatment, Raman, XPS, and TEM measurements were carried out for both the pristine and the acid-treated tubes (figure 1). The relatively increased $R$ value $\left(\mathrm{I}_{\mathrm{D}} / \mathrm{I}_{\mathrm{G}}\right.$, which is the intensity of the $\mathrm{D}$ band at $1325 \mathrm{~cm}^{-1}$ divided by the intensity of the $\mathrm{G}$ band at 1580 $\mathrm{cm}^{-1}$ ) of the acid treated tubes (figure 1(a)) indicates the effective generation of structural defects on the sidewall of the nanotubes [19]. XPS clearly supports the introduction of chemical moieties on the sidewall of the tubes via acid treatment (figure 1(b)). In the case of the pristine tubes, there are peaks at $285.5 \mathrm{eV}, 286.5 \mathrm{eV}$, and $287.5 \mathrm{eV}$ corresponding to $\mathrm{C}-\mathrm{C}$, $-\mathrm{C}-\mathrm{O}-$, and $-\mathrm{C}=\mathrm{O}$ bonds, respectively. However, a newly observed peak at $288.9 \mathrm{eV}$ in the acid-treated tubes indicates the introduction of -COO- groups [20]. TEM observations also support the effectiveness of acid treatment for the introduction of defects and chemical moieties (figure 1(c)) since the relatively long straight fringes are easily transformed into short corrugated fringes by generating structural defects preferentially on the sidewall of the tubes. 

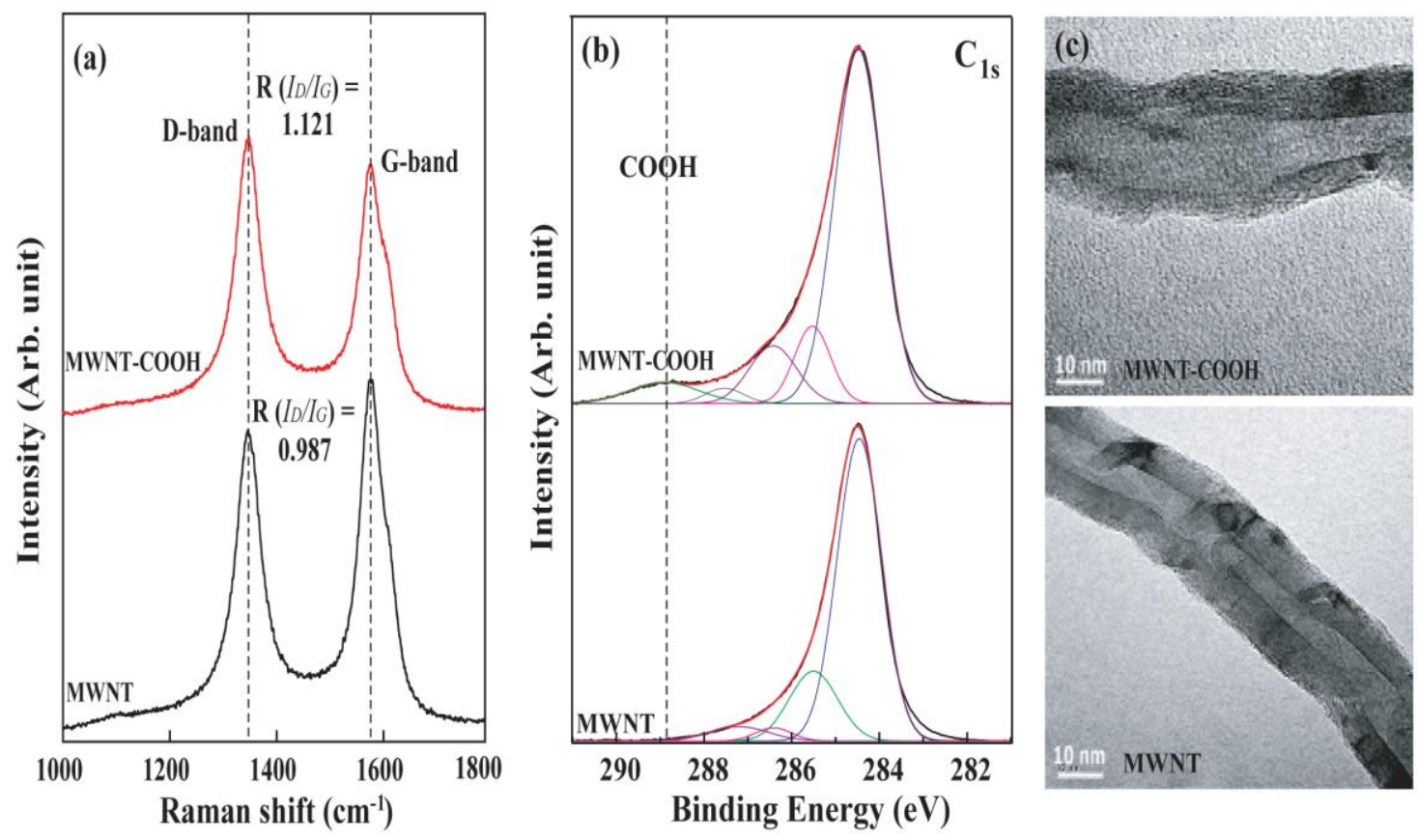

Fig.1. (a) Raman spectra, (b) C1 XPS spectra, and (c) TEM images of pristine and acid-treated multi-walled carbon nanotubes.

In order to compare the dispersion state of carbon nanotubes within the polyurethane, we performed detailed TEM and AFM observations on the spin-coated samples (figure 2). From the TEM images (figure 2(a)), in-situ and cross-linking polymerized composites have more black dots and lines, indicating the homogeneous dispersion of carbon nanotubes, while the conventionally blended composite has aggregated tubes due to poorly dispersed tubes. These results can be explained by the interaction between the nanotubes and the polyurethane molecules [16, 21]. We also confirmed the enhanced dispersion structure of the tubes in cross-linking polymerized composites, based on the presence of bright spots from the topographical AFM mapping images(figure 2(b)) and current AFM images (figure 2(c)) [22]. 

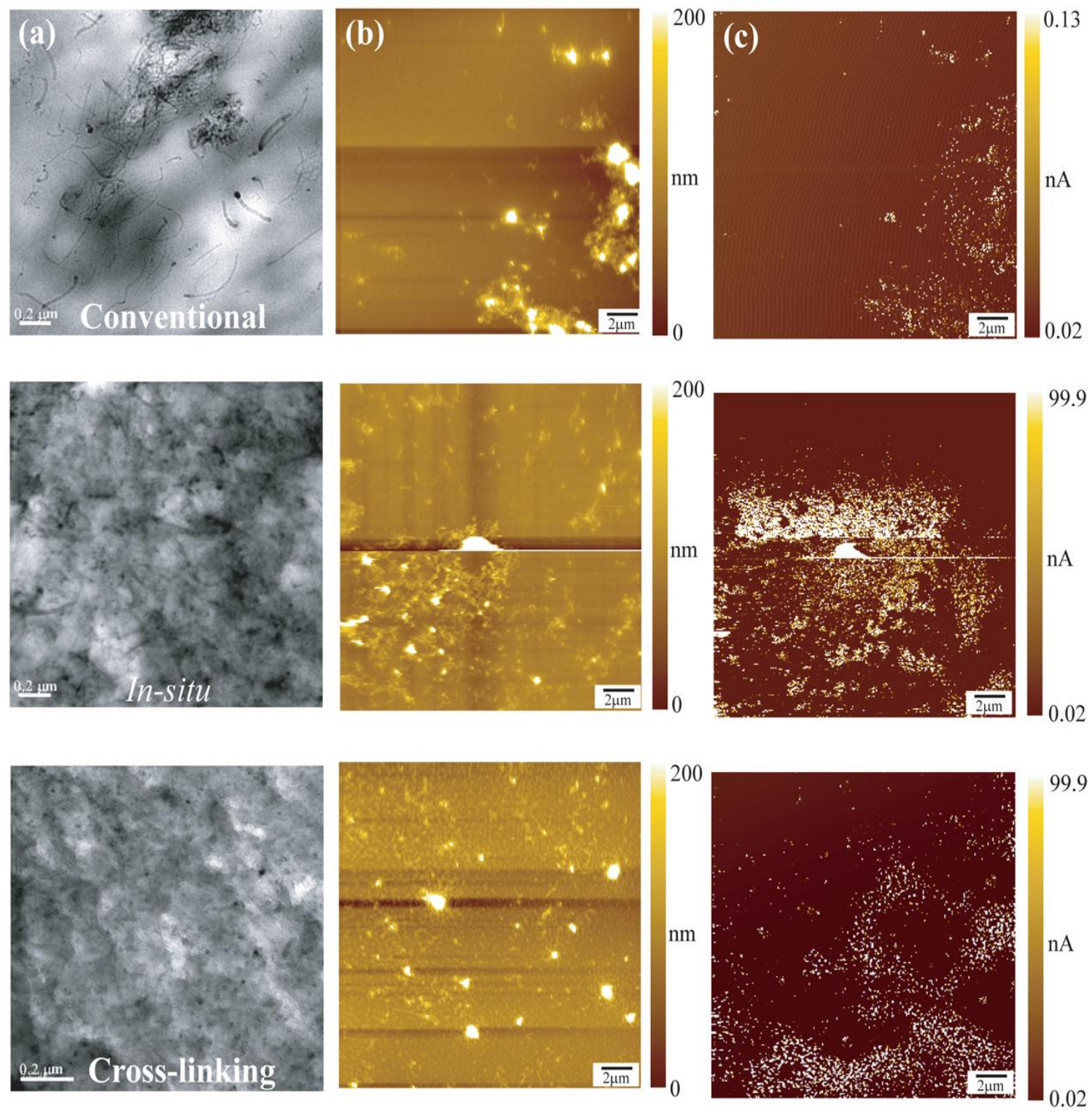

Fig.2. (a) TEM images, and (b) topographical and (c) current mapping AFM images for the polyurethane composites, prepared by conventional blending, in-situ, and cross-linking polymerization.

From the above results, it was concluded that cross-linking polymerization is the 
optimal way to disperse the tubes homogeneously within the polyurethane matrix. Here we suggest a blocking effect of the cross-linked polymer chains with respect to the tubes, where the covalently bonded tubes/polymer chains effectively prevent the re-aggregation of the tubes, thus fixing the tubes within the polyurethane matrix in a homogeneous distribution. Regarding the polyurethane composites prepared with in-situ polymerization, it is possible for the nanotubes to reorganize themselves in an aggregated form since they are physically dispersed within the polyurethane matrix. Consequently, we achieved the homogeneous dispersion of covalently fixed carbon nanotubes within the polyurethane matrix through the judicious selection of cross-linking polymerization (figure 3(a)).

It is noteworthy that the differences in the dispersion structure of the tubes depending on the polymerization method lead to differences in the physical properties of the resultant composites. For example, carbon nanotubes have been utilized as highly conductive routes for the purpose of increasing the electrical conductivity of various composites. In the case of materials possessing conductive shape memory $[16,21]$, the variation of the surface temperature due to electric heating caused by the application of voltage is closely related to the dispersion structure of the tubes within the polymer matrix. In this regard, we compared the electroactive shape memory of composites prepared by conventional blending, in-situ and cross-linking polymerization (figure 3(b) and 3(c)). A twofold improvement of the thermomechanical properties of the samples prepared with in-situ and cross-linking polymerization which has an elongation of up to $200 \%$ was observed in comparison to that of the conventionally blended sample (figure 3(b)). When the samples were elongated up to $200 \%$, they were unloaded after quenching to room temperature using liquid nitrogen. Finally, the 
change in length of the samples due to the applied electric voltage of $40 \mathrm{~V}$ was measured in order to obtain shape recovery (figure 4). The highest shape retention (92\%) and shape recovery (95\%) were observed for the composite prepared with cross-linking polymerization (figure 3(c)), whereas a shape recovery of $92 \%$ and $83 \%$ was obtained for the composites prepared by in-situ polymerization and conventional blending, respectively.

(a)
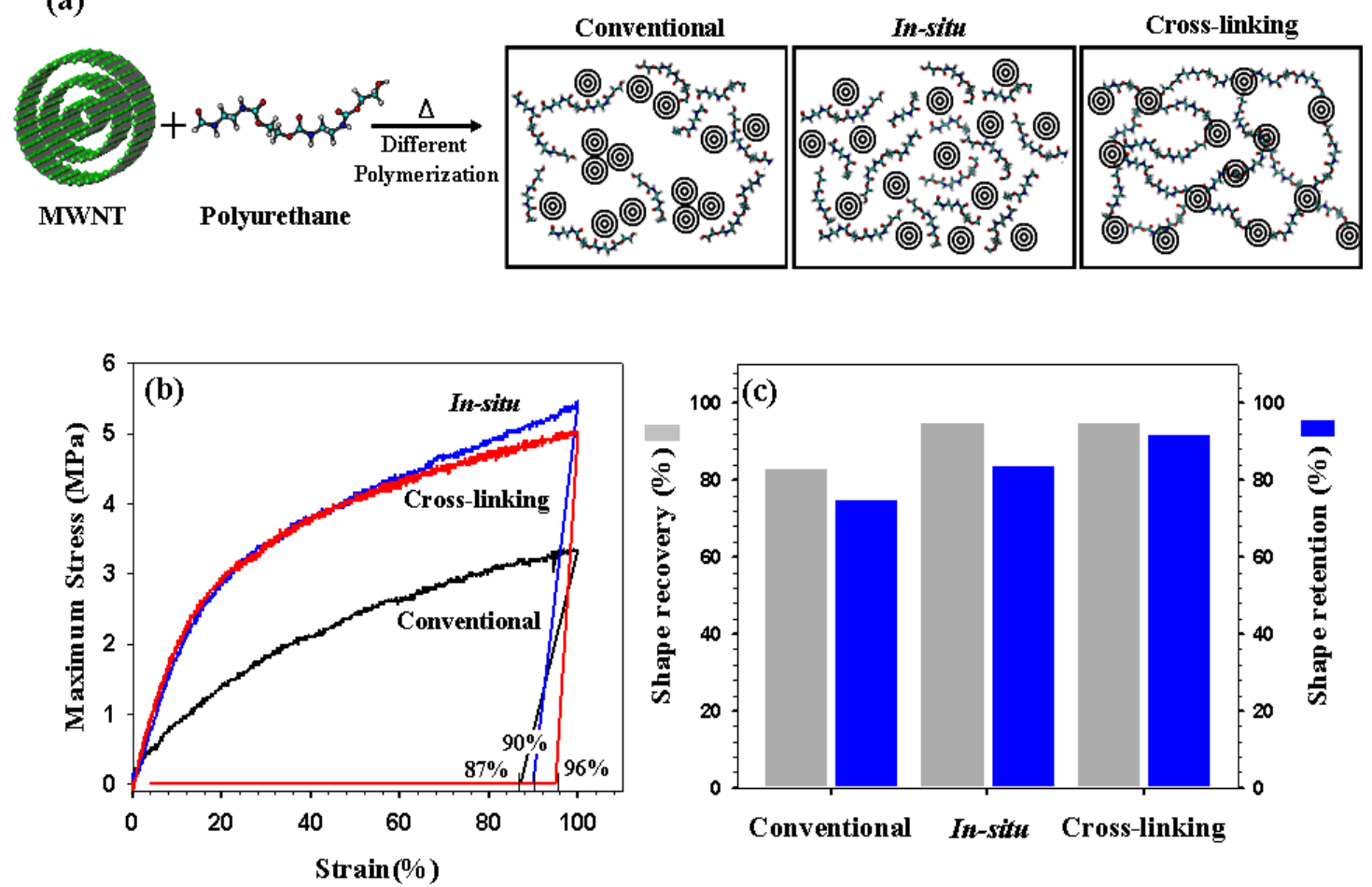

Fig.3. (a) A schematic representation of the controlled dispersed structures of the tubes within the polymer matrix, (b) thermomechanical properties curves (200\% elongation at $50^{\circ} \mathrm{C}$ ), and (c) shape recovery and shape retention (quenching/unloading and subsequently applying an electric voltage of $40 \mathrm{~V}$ ) for the polyurethane composites prepared by conventional blending, in-situ, and cross-linking polymerization. 


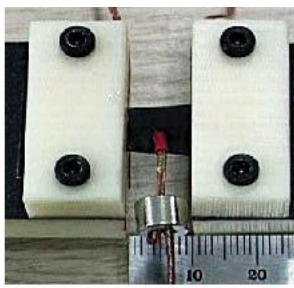

Original Shape $(100 \mathrm{~mm})$

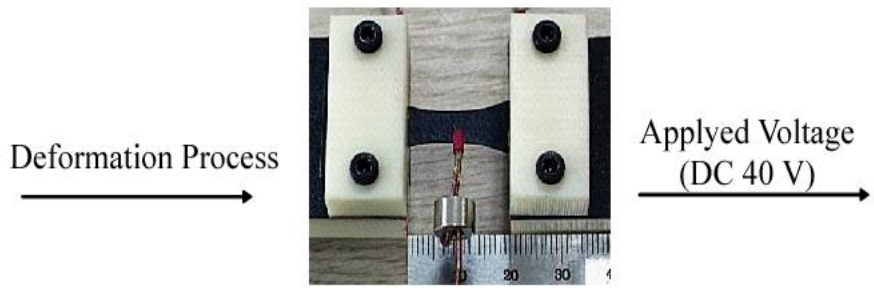

Shape Fixed $(200 \mathrm{~mm})$

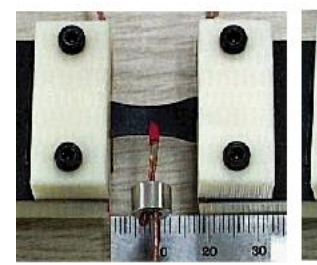

$10 \mathrm{sec}$

(180mm)

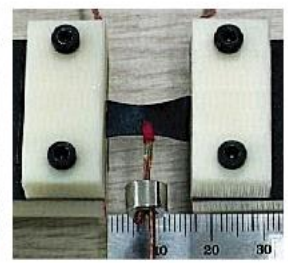

$15 \mathrm{sec}$

(170mm)

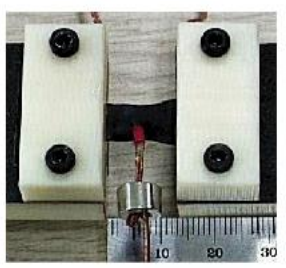

$25 \mathrm{sec}$

(140mm)

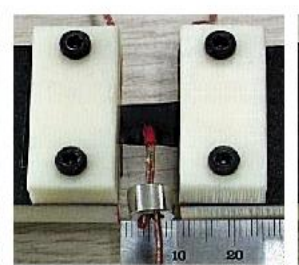

$35 \mathrm{sec}$

(110mm)

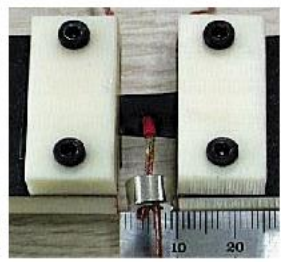

$50 \mathrm{sec}$

$(100 \mathrm{~mm})$

Fig.4. Pictures describing the electroactive shape recovery behavior of MWCNTs (4 wt\%)-reinforced polyurethane-derived composite. The sample undergoes the transition from temporary shape (original length: 100mm) to permanent (deformation length: $200 \mathrm{~mm}$ ) within 60 seconds when a constant voltage of $40 \mathrm{~V}$ was applied.

The variation of the surface temperature of the conductive polymer composites due to the application of electric power can be used as an effective indicator of the dispersion structure of the nanotubes (figure 5(a)). By applying an electric voltage of $40 \mathrm{~V}$ to the composites, we measured the temperature as a function of time. It is noteworthy that the surface temperature increased up to $100^{\circ} \mathrm{C}$ within 60 seconds in the composite prepared by cross-linking polymerization. However, the conventionally blended composite reached an equilibrium temperature of $38^{\circ} \mathrm{C}$ within 20 seconds. Interestingly, the patterns of the increasing surface temperature are very similar to those of the shape 
recovery curves (figure 5(b)) for all samples. The sample prepared by cross-linking polymerization exhibits the highest increase rate for shape recovery with respect to time, and soon reaches an equilibrium shape recovery state. From these results, it is expected that the variations of the surface temperature can be utilized as an indirect indicator for evaluating the electroactive shape recovery behavior of our conductive polyurethane composites since both properties are strongly influenced by the dispersion structure of the tubes.
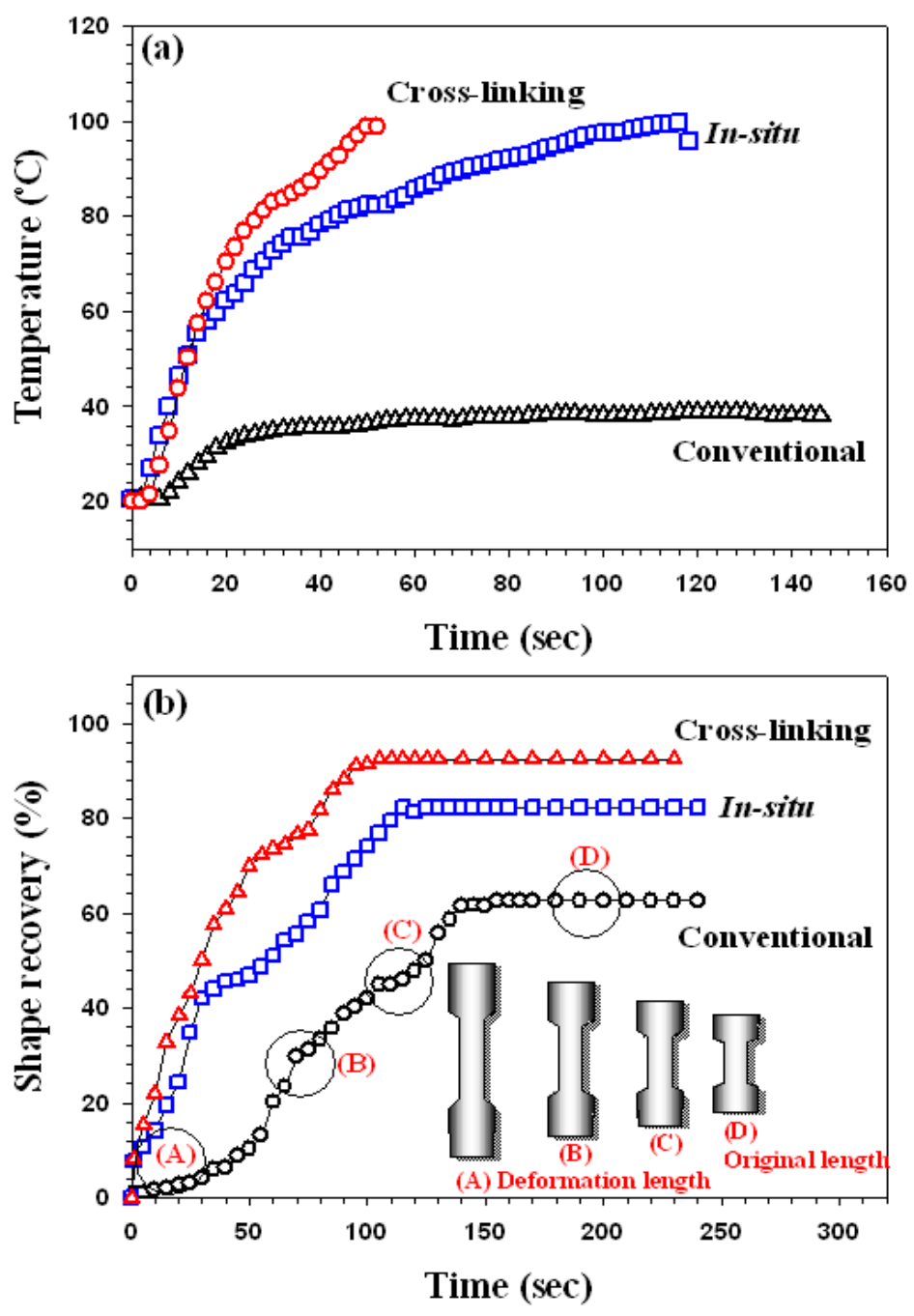
Fig.5. The fluctuations of the surface temperature and the shape recovery for the polyurethane composites prepared by conventional blending, in-situ and cross-linking polymerization for a voltage of $40 \mathrm{~V}$.

\section{Conclusions}

In conclusion, we succeeded in controlling the dispersion structure of the tubes in polyurethane composites filled with nanotubes by using conventional blending, in-situ, and cross-linking polymerization methods. We found that the optimal dispersion structure of the tubes within the polyurethane matrix is achieved by using chemically modified carbon nanotubes with carboxyl groups on their walls together with the cross-linking polymerization method. The covalently linked nanotubes/polyurethane hybrid structure effectively prevents the re-aggregation of the tubes within the polymer matrix. The composites prepared by cross-linking polymerization exhibited superior mechanical and shape memory properties in comparison to the ones prepared with in-situ polymerization and conventional blending. We found that both the surface temperature and the shape recovery with respect to the applied electric voltage were strongly influenced by the dispersed structure of the tubes in polyurethane composites filled with nanotubes. Both the homogeneous dispersion of carbon nanotubes within polyurethane and the covalent bonds between them are critical conditions for achieving high-performance shape memory.

\section{Acknowledgements}


This work was supported by the SRC/ERC Program of MOST/KOSEF (R11-2005-065) by the Korea government. We acknowledge the support from the CLUSTER Project (second stage) and a Grant-in-Aid (No. 20510096) from the Ministry of Education, Culture, Sports, Science and Technology of Japan.

\section{Reference}

[1] Dresselhaus M S, Dresselhaus G, Eklund P C. Science of Fullerene and Carbon Nanotubes. Academic: New York; 1995.

[2] Ajayan P M, Schadler L S, Giannaris C, Rubio A. Single-walled carbon nanotube-polymer composites: strength and weakness. Adv Mater 2000;12(10):750-53.

[3] Du F, Fischer J E, Winey K I. Coagulation method for preparing single-walled carbon nanotube/poly(methyl methacrylate) composites and their modulus, electrical conductivity, and thermal stability. J Polym Sci Part B: Polym Phys $2003 ; 41: 3333-38$

[4] Hill D E, Lin Y, Rao A M, Allard L F, Sun Y-P. Functionalization of carbon nanotubes with polystyrene. Macromolecules 2002;35:9466-71.

[5] Tang B Z, Xu H. Preparation, alignment, and optical properties of soluble poly(phenylacetylene)-wrapped carbon nanotubes. Macromolecules 1999;32:2569-76.

[6] O’Connell M J, Boul P, Ericson L M, Huffman C, Wang Y, Haroz E, et al. Reversible water-solubilization of single-walled carbon nanotubes by polymer wrapping. Chem Phys Lett 2001;342:265-71.

[7] Barraza H J, Pompeo F, O’Rear E A, Resasco D E. SWNT-filled thermoplastic and 
elastomeric composites prepared by miniemulsion polymerization. Nano Lett 2002;2:797-02.

[8] Dalton A B, Collins S, Muñoz E, Razal J M, Ebron V H, Ferraris J P, et. al. Super-tough carbon-nanotube fibers. Nature 2003;423:703.

[9] Regev O, ElKati P N B, Loos J, Koning C E. Preparation of conductive nanotube-polymer composites using latex technology. Adv Mater 2004;16(3):248-51.

[10] Viswanathan G, Chakrapani N, Yang H C, Wei B Q, Chung H S, Cho K W, et al. Single-step in situ synthesis of polymer-grafted single-wall nanotube composites. J Amer Chem Soc 2003;125:9258-59.

[11] Velasco-Santos C, Martinez-Hernández A L, Fisher F T, Ruoff R, Castaño V M. Improvement of Thermal and Mechanical Properties of Carbon Nanotube Composites through Chemical Functionalization. Chem Mater 2003;15:4470-75.

[12] Geng H Z, Rosen R, Zheng B, Shimoda H, Fleming L, Liu J, Zhou O. Fabrication and Properties of Composites of Poly(ethylene oxide) and Functionalized Carbon Nanotubes. Adv Mater 2002;14(19):1387-90.

[13]Nandivada H, Jiang X, Lahann J. Click Chemistry: Versatility and Control in the Hands of Materials Scientists. Adv Mater 2007;19:2197-08.

[14]Chen R J, Zhang Y, Wang D, Dai H. Noncovalent Sidewall Functionalization of Single-Walled Carbon Nanotubes for Protein Immobilization. J Amer Chem Soc 2001;123:3838-39.

[15] Gao J, Itkis M E, Yu A, Bekyarova E, Zhao B, Haddon R C. Continuous spinning of a single-walled carbon nanotube-nylon composite fiber. J Amer Chem Soc $2005 ; 127: 3847-54$. 
[16] Jung Y C, Sahoo N G, Cho J W. Polymeric nanocomposites of polyurethane block copolymers and functionalized mult-walled carbon nanotubes as crosslinkers. Macromol Rapid Commun 2006;27:126-31.

[17]Lee B S, Chun B C, Chung Y C, Sul K L, Cho J W. Structure and thermomechanical properties of polyurethane block copolymers with shape memory effect. Macromolecules 2001;34:6431-37.

[18]Leng J S, Lv H, Liu Y J, Du S Y. Electroactive shape-memory polymer filled with nanocarbon particles and short carbon fibers. Appl Phys Lett 2007;91:144105-3.

[19] Knight D S, White W B. J. Characterization of diamond films by Raman spectroscopy. Mater Res 1989;4(2):385-93.

[20] Ago H, Kugler T, Cacialli F, Salaneck W R, Shaffer M S P, Windle A H, et al. Work functions and surface functional group of multiwall carbon nanotubes. $\mathbf{J}$ Phys Chem B 1999;103:8116-21.

[21]Li F, Qi L, Yang J, Xu M, Luo X, Ma D. Polyurethane/conducting carbon black composites: structure, electric conductivity, strain recovery behavior, and their relationships. J Appl Polym Sci 2000;75:68-77.

[22] Bliznyuk V, Singamaneni S, Kattumenu R, Atashbar M. Surface electrical conductivity in ultrathin single-wall carbon nanotube/polymer nanocomposite films. Appl Phys Lett 2006;88:164101-3. 